\title{
FREQUENCY AND DURATION OF OXIMETER DROP-OUTS IN THE NICU: AN OBSERVATIONAL STUDY
}

\author{
Thomas Bachman, Karel Roubik \\ Department of Biomedical Technology, Faculty of Biomedical Engineering, \\ Czech Technical University in Prague, Kladno, Czech Republic
}

\begin{abstract}
Oximeters used for continuous monitoring experience periods with no signal. This $\mathrm{SpO}_{2}$ drop-out is widely acknowledged and its causes generally understood. This is a prospectively designed analysis of an existing database with the aim of characterizing drop-outs as experienced in the neonatal ICU. The data reflects 116 days of monitoring in seven tertiary care neonatal ICUs in 6 countries. From the evaluation of 1,396 drop-outs we found that typically the time was minimal with missing $\mathrm{SpO}_{2}$ data, and the episodes were short (median 2.79 minutes per day IQR 0.17-76, median 22 seconds IQR: 15-37, respectively). During about half of the days there were no prolonged dropouts (1 minute or longer), even so half of the total time spent with no SpO $\mathrm{S}_{2}$ data were in prolonged episodes (median length 110 seconds IQR 85-150). The predominate factor associated with excessive drop-out time was the number of prolonged episodes rather than their duration. We concluded that the impact of drop-outs during manual control of inspired oxygen primarily impact alarm fatigue, but that during automatic $\mathrm{FiO}_{2}$ control they could have an important impact. The relative effectiveness of the fallback strategies of these automatic control systems ought to be evaluated.
\end{abstract}

\section{Keywords}

oximeter, oxygen saturation, neonatal ICU, $\mathrm{SpO}_{2}$ drop-out

\section{Background}

Continuous monitoring of neonatal oxygenation using the pulse oximeter is an essential tool in the neonatal intensive care unit (NICU). Relatively small changes in overall exposure to high and low extremes of peripheral oxygen saturation $\left(\mathrm{SpO}_{2}\right)$ levels have been shown to have significant impact on neonatal outcome [1, 2]. In addition, exposure to extreme levels have been shown to be primarily episodic with episodes of 1 minute or longer contributing most significantly to bad outcomes [3].

While an essential tool, the use of the pulse oximeter is problematic. False alarms are common and known to create significant alarm fatigue [4]. It is well documented that this leads to risks of frequent disregard for alarm policy and $\mathrm{SpO}_{2}$ targeting guidelines [5].

The reasons for these false readings, that is artifact, are understood. They are primarily caused by motion, low regional perfusion and loss of sensor integrity. Even though the $\mathrm{SpO}_{2}$ is calculated based on an average of 3-15 pulses, there are still occasions when no reading is presentable. In this occasion it is said that the $\mathrm{SpO}_{2}$ reading “drops out”. While this is a common occurrence, we are unaware of any reports characterizing drop-outs in the NICU.

Much of the practice of adjusting the inspired oxygen using pulse oximetry is applied pragmatically by the attending nurse. General good practice would dictate that oxygen adjustments should be made judiciously, with a goal of avoiding responses to false positives or providing unnecessary extra oxygen in response to a short transient physiological perturbation. Periods of drop-outs are treated in the same manner. However, new neonatal ventilators offering nearly continuous automated adjustment of $\mathrm{FiO}_{2}$ based on monitored $\mathrm{SpO}_{2}$ have been shown to be quite effective [6]. International standards do require fail-back modes for periods of missing or unreliable signals. "Fall-back" being the preferred term to the commonly used, "failsafe”.

As a part of our work to improve alarm and oxygenation control practices, we are evaluating a large database of $\mathrm{SpO}_{2}$ recordings from NICUs. As part of this EPI-SAT project, we supposed it important to characterize the frequency and duration of $\mathrm{SpO}_{2}$ dropouts as they occur in real NICUs. 


\section{Methods}

The EPI-SAT database includes 116 days of continuous $\mathrm{SpO}_{2}$ data from 58 significantly preterm infants receiving respiratory support and supplemental oxygen. These infants all participated in studies of $\mathrm{SpO}_{2}$ control and are from seven neonatal ICU's in six countries [7, 8]. Consent was received for participation in the studies and all the data in the EPI-SAT database is de-identified.

$\mathrm{SpO}_{2}$ readings were collected every 5 seconds, by a data-logger attached to the digital output of a mechanical ventilator (AVEA-CLiO2, Vyaire Medical, Irvine, CA, USA). The oximeter used Masimo SET technology (Masimo, Irvine, CA, USA) with averaging set at 8 seconds. Purpose built software (MatLab, MathWorks, MA, USA) evaluated drop-out episodes and parsed them into 5 duration categories: $<5 \mathrm{~s}, 5$ to $<30 \mathrm{~s}, 30$ to $<60 \mathrm{~s}, 60$ to $<120 \mathrm{~s}, 120$ to $<600 \mathrm{~s}$. Episodes of 10 minutes or longer were excluded as likely procedural interventions, rather than drop-outs. The latter two categories (length of 1 minute or greater) were defined as being relevant to $\mathrm{SpO}_{2}$ control $\left(\mathrm{FiO}_{2}\right.$ adjustment). High outliers were identified based on a standard nonparametric method. Specifically the high outlier threshold was the interquartile span plus the seventy-fifth percentile.

Differences between the outlier and non-outlier groups were compared with the Mann-Whitney U-test. Differences among the 5 episode length categories were compared with the Kruskal-Wallis test with Dunn's procedure for pairwise comparisons. A twotailed $\mathrm{p}<0.05$ was considered statistically significant. Statistical tests were conducted with XLSTAT v19.03 software (Addinsoft, Paris, France).

\section{Results}

We identified and analyzed 1,396 drop-outs during the 116 recorded days, that is 12 per day. The typical total time with missing $\mathrm{SpO}_{2}$ readings (drop-outs) was minimal (median 2.79 minutes/day, range 0.17-76), obviously skewed. The actual median duration of individual drop-outs was 22 seconds (IQR: 15-37).

The distribution of drop-out time among the five duration categories was: $7 \%$ for $<5$ s, $24 \%$ for 5 to $<30$ s, $21 \%$ for 30 to $<60$ s, $21 \%$ for 60 to $<120$ s, and $29 \%$ for 120 s or greater. Thus about half of the dropout time was deemed, according to our prospective definition, relevant to $\mathrm{SpO}_{2}$ control. Details of the variation among the duration categories are tabulated in Table 1. During 59 of the 116 days, there were no prolonged drop-outs. When present, the median duration of these prolonged drop-outs was almost 2 minutes (110 s, IQR 85-150).
Table 1: Total Drop-Out time in each duration category (minutes/day).

\begin{tabular}{lrcr}
\hline Category & \multicolumn{3}{c}{ Duration } \\
\cline { 2 - 4 } & $\mathrm{n}(\mathrm{n} /$ day $)$ & Median (IQR) & Range \\
\hline$<5 \mathrm{~s}$ & $485(4.2)$ & $0.17(0.08-0.25)$ & $0-18$ \\
5 to $<30 \mathrm{~s}$ & $572(4.9)$ & $0.83(0.42-1.33)$ & $0-29$ \\
30 to $<60 \mathrm{~s}$ & $195(1.7)$ & $0.58(0-1.35)$ & $0-15$ \\
60 to $<120 \mathrm{~s}$ & $89(0.8)$ & $0(0-1.35)$ & $0-16$ \\
120 to $<600 \mathrm{~s}$ & $55(0.5)$ & $0(0-2.0)$ & $0.2-32$ \\
Total & $1396(12)$ & $2.79(1.50-5.04)$ & $0.17-76$ \\
\hline
\end{tabular}

The differences in the frequency of and total length of time among each of the 5 categories are statistically significant $(p<0.001)$. The frequency of episodes between each categories were all statistically significantly different $(p<0.05)$, except between the two longest. The time in the shortest category was not significantly different than in the two longest categories. Other differences were significant $(\mathrm{p}<0.05)$.

Excessive drop-out time (outliers with >8.6 minutes of drop-out in a day) occurred in only 14 of the 116 days. In this group of outliers the median duration of time without $\mathrm{SpO}_{2}$ readings was 16 minutes (IQR 1131 ), significantly longer than the nominal group (2 minutes (IQR 1-4), $\mathrm{p}<0.001$ ). The predominant factor in the difference between the two categories was the number of prolonged episodes, rather than their duration. The median duration of prolonged drop-out episodes in the outlier drop-out group was $134 \mathrm{~s}$ (IQR 8-147) and was not significantly different from nominal group: $100 \mathrm{~s}$ (IQR 80-145). In contrast, the median rates of prolonged drop-outs per day were 4 (IQR 3.259.25) and 0 (IQR 0-1.0) respectively, and the difference was significant $(\mathrm{p}<0.001)$. Other details of the difference in the frequency of prolonged drop-outs are tabulated by outlier category in Table 2 .

Table 2: Frequency of Episodes 1 minute or longer (n/24 hours).

\begin{tabular}{lccc}
\hline Category & $\mathrm{n}$ & Median (IQR) & Range \\
\hline typical & 102 & $0(0-1)$ & $0-3$ \\
outliers & 14 & $4(3.25-9.25)$ & $2-14$ \\
\hline
\end{tabular}

The differences are statistically significant $(\mathrm{p}<0.001)$.

\section{Discussion}

In this group of very preterm infants who required respiratory support and supplemental oxygen, oximeter dropouts were common, that is one every few hours. Furthermore, the total time without $\mathrm{SpO}_{2}$ readings was relatively low and the typical duration of each episode relatively short. Only about half the days saw one or more prolonged drop-out, and only $12 \%$ of the days experienced an atypically high amount of time with 
drop-outs. We also found that in the case of the latter the total number of prolonged episodes was much higher.

The best practices for manual adjustment of inspired oxygen call for the nurse to observe the situation and only make adjustments after assessing the situation with the oximeter alarms and alerts as well as the infant. Response to a perturbation in $\mathrm{SpO}_{2}$ quicker than 1-2 minutes, except in the case of an emergency, would be considered too fast. One study did look at the effecttiveness of $\mathrm{SpO}_{2}$ control with two manual strategies; attentive (45-75 s) and observative (130-195 s) [9]. They found that faster was better. Nevertheless, in routine care the risk of excessive overshoot in oxygen could only be mitigated with a dedicated operator as used in the study, so caution was advised. We found only a small percentage of the days of care were associated with frequent prolonged episodes, and most of those were shorter than 2 minutes. Thus, it would seem that the practice of only considering a change to $\mathrm{FiO}_{2}$ if an alarm condition persists for 1-2 minutes mitigates most of the concern about what adjustment should be made during periods of drop out. Nevertheless, false alarms from artifacts, including dropouts, are a constant irritant and a source of the risk associated with alarm fatigue [10]. Further, one careful study did evaluate the likely $\mathrm{SpO}_{2}$ reading following drop-out and Lim et al. concluded that blind adjustment to $\mathrm{FiO}_{2}$ should not be made during drop-out [11].

Neonatal respiratory support systems that automatically adjust inspired oxygen based on $\mathrm{SpO}_{2}$ (Auto$\mathrm{FiO}_{2}$ ) have become available from a number of companies and are being widely adopted [12]. They continuously monitor $\mathrm{SpO}_{2}$ and many make adjustments every second as necessary.

The long established international standard for physiological control systems require a fall-back function when the signal to the control algorithm is unavailable [13]. Because of the continuous $\mathrm{SpO}_{2}$ signal into the controller, every drop-out no matter how short has impact and must be addressed. In general the approach of these Auto- $\mathrm{FiO}_{2}$ controllers is not documented. One Auto- $\mathrm{FiO}_{2}$ system stops the auto function when the input is unavailable, or if there is a major drop in $\mathrm{SpO}_{2}$, and requires manual intervention by the clinician. Another system ignores short drop-out periods but if they persist changes the $\mathrm{FiO}_{2}$ to a fall-back level based on several previous $\mathrm{FiO}_{2}$ metrics. No fall-back approach is perfect, but they all ought to be compared to standard manual care. One such study has just finished and the results are promising [personal communication]. Predictably, it appears that following drop-outs Auto- $\mathrm{FiO}_{2}$, when compared to manual control, reduces the likelihood of hypoxemia, and increases the likelyhood of normoxemia but also hyperoxemia. Auto-FiO systems have been shown in clinical studies to be safe and effective [6]. However, suboptimal fall-back approaches, as suggested by the work of Lim [11], might detract from their potential effectiveness, and potential- ly pose risks. These might include excessive overshoot from hypoxemia or swings between hypoxemia and hyperoxemia.

Our analysis has some limitations. First, each dropout was treated as an independent event. It is likely that a period of several minutes of intermittent short dropouts would have the same clinical impact on manual and auto control as one long episode. Although the total time with missing $\mathrm{SpO}_{2}$ values was relatively small, the prevalence of clusters of drop-out ought to be explored. Second, while this is to our knowledge the first report of the characterization of drop-outs, it reflects the experience of 58 neonates, one study with a different oximeter suggests a higher frequency of drop-outs [11], which could reflect a different group of infants or a different averaging of the oximeter. Additional studies to broaden these findings and these results are warranted.

\section{Conclusion}

We confirmed that oximeter drop-outs probably do not generally impact clinical decision making during manual titration of inspired oxygen. While their prevalence is limited, and they are of short duration, they do contribute to false alarms and the potential for alarm fatigue as an acknowledged risk factor. In contrast, we suggest their prevalence would have an impact on the effectiveness during automated control of inspired oxygen. For this reason the fall-back approaches of commercially available systems ought to be documented and evaluated.

\section{Acknowledgement}

The work was supported by a research grant SGS20/202/OHK4/3T/17 of the Czech Technical University in Prague.

\section{Author contributions}

The first author (TB) provided the concept for the study, and wrote the first draft of the manuscript. The second author (KR) coded the data extraction software. Both authors critically reviewed the data and the manuscript.

\section{References}

[1] Askie LM, Darlow BA, Finer N, et al. Association between oxygen saturation targeting and death or disability in extremely 
preterm infants in the Neonatal Oxygenation Prospective Metaanalysis Collaboration. JAMA. 2018;319(21):2190-201. DOI: 10.1001/jama.2018.5725

[2] Di Fiore J, Bloom J, Orge F, et al. A higher incidence of intermittent hypoxemic episodes is associated with severe retinopathy of prematurity. J Pediatr 2010;157(1):69-73. DOI: 10.1016/j.jpeds.2010.01.046

[3] Poets C, Roberts R, Schmidt B, et al. Association between intermittent hypoxemia or bradycardia and late death or disability in extremely preterm infants. JAMA 2015;314(6): 595-603. DOI: 10.1001/jama.2015.8841

[4] Ketko AK, Martin CM, Nemshak MA, et al. Balancing the tension between hyperoxia prevention and alarm fatigue in the NICU. Pediatrics 2015;136(2):496-504. DOI: 10.1542/peds.2014-1550

[5] Hagadorn JI, Sink DW, Buus-Frank ME, et al. Alarm safety and oxygen saturation targets in the Vermont Oxford Network iNICQ 2015 collaborative. J Perinatol 2017;37(3):270-6. DOI: 10.1038/jp.2016.219

[6] Mitra S, Singh B, El-Naggar W, McMillan DD. Automated versus manual control of inspired oxygen to target oxygen saturation in preterm infants: a systematic review and metaanalysis. J Perinatol. 2018;38(4):351-60. DOI: 10.1038/s41372-017-0037-z

[7] van Kaam, A.H., Hummler H, Wilinska M, et al. Automated versus manual oxygen control with different saturation targets and modes of respiratory support in preterm infants. J Pediatr 2015;167(3):545-50. DOI: 10.1016/j.jpeds.2015.06.012

[8] van den Heuvel ME, van Zanten HA, Bachman T, et al. Optimal target range of closed-loop inspired oxygen support in preterm infants: a randomized controlled study. J Pediatr 2018;197:3641. DOI: $10.1016 / j . j p e d s .2018 .01 .077$

[9] Wilinska M, Bachman T, Swielinski J, et al, Quicker response results in better $\mathrm{SpO}_{2}$ control - a comparison of $3 \mathrm{FiO}_{2}$-titration strategies in ventilated preterm infants. Ann Agric Environ Med. 2015;22(4):708-12. DOI: $\underline{10.5604 / 12321966.1185781}$
[10] Johnson KR, Hagadorn JJ, Sink DW. Alarm Safety and Alarm Fatigue. Clin Perinatol 2017;44:713-28. DOI: 10.1016/j.clp.2017.05.005

[11] Lim K, Wheeler $\mathrm{K}$, Jackson $\mathrm{H}$, et al. Lost without trace: oximetry signal dropout in preterm infants. Arch Dis Child, 2015;100(5):435-8. DOI: 10.1136/archdischild-2014-308108

[12] Wilinska M, Skrzypek M, Bachman T, et al. Using the automated $\mathrm{FiO}_{2}-\mathrm{SpO}_{2}$ control in neonatal intensive care units in Poland: a preliminary report. Developmental Period Medicine 2015;XIX,3(216).

[13] IEC 60601-1-10: Medical electrical equipment - Part 1-10: General requirements for basic safety and essential performance - Collateral standard: Requirements for the development of physiologic closed-loop controllers. The International Electrotechnical Commission, 2007.

Thomas E. Bachman MSc. Department of Biomedical Technology Faculty of Biomedical Engineering Czech Technical University in Prague nám. Sítná 3105, CZ-272 01 Kladno

$\&$

Economedtrx www.medtrx.org

Lake Arrowhead, CA, USA, 92352-1269

E-mail: tbachman@me.com thomas.bachman@fbmi.cvut.cz Phone: +1 909 337-0828 